\title{
A localized Erdős-Kac theorem
}

\author{
Anup B. Dixit and M. Ram Murty
}

Dedicated to the memory of Srinivasa Ramanujan

\begin{abstract}
Let $\omega_{y}(n)$ be the number of distinct prime divisors of $n$ not exceeding $y$. If $y_{n}$ is an increasing function of $n$ such that $\log y_{n}=o(\log n)$, we study the distribution of $\omega_{y_{n}}(n)$ and establish an analog of the Erdős-Kac theorem for this function. En route, we also prove a variant central limit theorem for random variables, which are not necessarily independent, but are well approximated by independent random variables.
\end{abstract}

Keywords. prime divisors, Erdős-Kac theorem, central limit theorem

2010 Mathematics Subject Classification. 11N25, 11N64, 11K65, 60F05

\section{Introduction}

Let $\omega(n)$ denote the number of distinct prime divisors of the natural number $n$. It is an interesting question to study how $\omega(n)$ is distributed as we vary $n \leq x$. The average value of $\omega(n)$ for $n \leq x$ can be computed as

$$
\frac{1}{x} \sum_{n \leq x} \omega(n)=\frac{1}{x} \sum_{p \leq x} \sum_{\substack{n \leq x, p \mid n}} 1=\frac{1}{x} \sum_{p \leq x}\left[\frac{x}{p}\right]=\log \log x+O(1)
$$

as $x \rightarrow \infty$. In 1917, Hardy and Ramanujan [HR1917] proved that the normal order of $\omega(n)$ is $\log \log n$. More precisely, for any $\epsilon>0$, as $x \rightarrow \infty$, we have

$$
\#\{n \leq x \mid n \text { satisfies }|\omega(n)-\log \log n|>\epsilon \log \log n\}=o(x)
$$

A simplified proof of the Hardy-Ramanujan result was given by Turán [Tu34] in 1934, by considering the second moment of $\omega(n)$. He proved that

$$
\sum_{n \leq x}(\omega(n)-\log \log x)^{2} \ll x \log \log x
$$

Note that in the summand above, $\log \log x$ can be replaced with $\log \log n$, since they are very close in value for all but very small integers, owing to the slow growth of the function $\log \log x$. Thus, one can easily deduce (1.1). In fact, Hardy and Ramanujan [HR1917] showed that if $c(n) \rightarrow \infty$ as $n \rightarrow \infty$, then

$$
|\omega(n)-\log \log n| \leq c(n) \sqrt{\log \log n}
$$

for almost all $n \leq x$, no matter how slowly $c(n)$ grows.

Research of the first author was partially supported by a Coleman Postdoctoral Fellowship. Research of the second author was partially supported by NSREC Discovery grant.

We thank episciences.org for providing open access hosting of the electronic journal Hardy-Ramanujan Journal 
From a probabilistic perspective, inequality (1.2) is reminiscent of Chebycheff's inequality and inequality (1.3) suggested that perhaps $\omega$ was a "random variable" on the space of natural numbers with "mean" $\log \log n$ and "standard deviation" $\sqrt{\log \log n}$. So after Turán's paper appeared, M. Kac posed the question of finding the distribution of

$$
\frac{\omega(n)-\log \log n}{\sqrt{\log \log n}},
$$

as $n$ varies. He suggested that this distribution is perhaps Gaussian. Kac recalls with evident delight "If I remember it correctly I first stated (as a conjecture) the theorem on the normal distribution of the number of prime divisors during a lecture in Princeton in March 1939. Fortunately for me and possibly for Mathematics, Erdős was in the audience, and he immediately perked up. Before the lecture was over he had completed the proof, which I could not have done not having been versed in the number theoretic methods, especially those related to the sieve." This was the magical moment when probabilistic number theory was born (see page 24 of [El79b]).

Shortly after this romantic episode, Erdös and Kac announced their result, which is famously known as the Erdös-Kac theorem [EK40]. They proved that for any real numbers $a, b$

$$
\lim _{x \rightarrow \infty} \frac{1}{x} \#\left\{n \leq x \mid a \leq \frac{\omega(n)-\log \log n}{\sqrt{\log \log n}} \leq b\right\}=\frac{1}{\sqrt{2 \pi}} \int_{a}^{b} e^{-t^{2} / 2} d t .
$$

Thus, the quantity in (1.4) has the standard normal distribution. As noted, the original proof of Erdős and Kac used Brun's sieve and the central limit theorem. Alternate proofs of the Erdös-Kac theorem were given later using different methods by Selberg [Sel53], Halberstam [Hal55], Billingsley [Bi69] (using the method of moments which we adopt below) and Shapiro [Sha56].

In this paper, we are interested in the truncated function, $\omega_{y}(n)$, which denotes the number of distinct prime divisors of $n$ not exceeding $y$. We study the behaviour of $\omega_{y}(n)$ and derive an analog of the Erdős-Kac theorem for it. We first make some observations before stating the main result.

The average order of $\omega_{y}(n)$ can be computed as before:

$$
\frac{1}{x} \sum_{n \leq x} \omega_{y}(n)=\frac{1}{x} \sum_{n \leq x} \sum_{\substack{p \mid n, p \leq y}} 1=\frac{1}{x} \sum_{p \leq y}\left[\frac{x}{p}\right]=\log \log y+O(1)
$$

as $x, y \rightarrow \infty$. Also note that

$$
\begin{aligned}
\frac{1}{x} \sum_{n \leq x} \omega_{y}^{2}(n) & =\frac{1}{x}\left(\sum_{\substack{p, q \leq y, p \neq q}}\left[\frac{x}{p q}\right]+\sum_{p \leq y}\left[\frac{x}{p}\right]\right) \\
& =\sum_{p, q \leq y} \frac{1}{p q}-\sum_{p \leq y} \frac{1}{p^{2}}+\log \log y+O\left(\frac{\pi^{2}(y)}{x}\right) \\
& =(\log \log y)^{2}+O(\log \log y)+O\left(\frac{\pi^{2}(y)}{x}\right)
\end{aligned}
$$

where $\pi(y)$ denotes the number of primes $\leq y$. Hence, the second moment is given by

$$
\frac{1}{x} \sum_{n \leq x}\left(\omega_{y}(n)-\log \log y\right)^{2}=O(\log \log y)+O\left(\frac{\pi^{2}(y)}{x}\right),
$$


as $x, y \rightarrow \infty$. From the above observation, it is reasonable to predict that if $y$ is sufficiently smaller than $x$, then $\omega_{y}(n)$ is typically $\log \log y$. More generally, for any multiplicative function $f: \mathbb{N} \rightarrow \mathbb{Q}$, the second moment of $\omega_{y}(f(n))$ is computed in [MM84a, Theorem 3.1]. We can derive our theorem in a more general setting though we do not do so here for the sake of simplicity of exposition. The general investigation can be found in [MM84a] and [MM84b].

Let $\Omega$ be the set of positive integers. $P_{n}$ be the probability measure placing mass $1 / n$ for each $\{1,2, \cdots, n\}$. We prove the following version of the Erdös-Kac theorem for $\omega_{y}(n)$.

Theorem 1.1. Let $y_{n}$ be an increasing sequence of real numbers satisfying $y_{n} \rightarrow \infty$ as $n \rightarrow \infty$ and suppose

$$
\lim _{n \rightarrow \infty} \frac{\log y_{n}}{\log n}=0
$$

Then, for any real numbers $a, b$,

$$
\lim _{n \rightarrow \infty} P_{n}\left(m: a \leq \frac{\omega_{y_{n}}(n)-\log \log y_{n}}{\sqrt{\log \log y_{n}}} \leq b\right)=\frac{1}{\sqrt{2 \pi}} \int_{a}^{b} e^{-t^{2} / 2} d t .
$$

Truncated additive functions, such as $\omega_{y}(n)$, have been previously considered in the literature. The distribution of $\omega_{y}(n)$ as in Theorem 1.1 appears as a step towards establishing the Erdös-Kac theorem, for instance, see [EK40, Lemma 5], [Sha56, pg.428, formula (10)] and with more restrictive conditions on $y_{n}$ in [Bi69, formula (21)] and [Hal55, Theorem 2]. The aim of this paper is to highlight the study of localized additive functions on account of their independent interest.

\section{Preliminaries}

The proof of the main theorem relies on the method of moments theorem and the Lyapunov central limit theorem, which are stated below (see pp. 312 and pp. 342 Theorem 30.1 in [Bi79]).

Theorem 2.1. (Method of moments) Let $\mu$ be a probability measure on the line having finite moments

$$
\alpha_{k}=\int_{-\infty}^{\infty} x^{k} \mu(d x)
$$

for all positive integers $k$. If the power series

$$
\sum_{k=1}^{\infty} \frac{\alpha_{k} r^{k}}{k !}
$$

has a positive radius of convergence, then $\mu$ is the only probability measure with moments $\alpha_{1}, \alpha_{2}, \cdots$.

Theorem 2.2. (Lyapunov central limit theorem) For $i \in \mathbb{N}$, let $X_{i}$ be independent random variables, with mean $\mu_{i}$ and variance $\sigma_{i}^{2}$ respectively. Denote by $\mathfrak{s}_{n}^{2}=\sum_{i=1}^{n} \sigma_{i}^{2}$. If for some $\delta>0$, the Lyapunov condition

$$
\lim _{n \rightarrow \infty} \frac{1}{\mathfrak{s}_{n}^{2+\delta}} \sum_{i=1}^{n} E\left[\left|X_{i}-\mu_{i}\right|^{2+\delta}\right]=0
$$

is satisfied, then

$$
\frac{1}{\mathfrak{s}_{n}} \sum_{i=1}^{n}\left(X_{i}-\mu_{i}\right) \rightarrow N(0,1)
$$

where $N(0,1)$ denotes the standard normal distribution, with mean 0 and variance 1. 


\section{A generalized central limit theorem}

Towards the proof of Theorem 1.1, we first establish a central limit theorem for random variables, which are not necessarily independent.

Let $f$ be a positive non-decreasing function on positive integers, such that as $n$ tends to infinity, $f(n)$ tends to infinity and

$$
\log f(n)=o(\log n)
$$

We prove the following.

Theorem 3.1. For $i \in \mathbb{N}$, let $X_{i}$ be independent random variables, taking bounded values and satisfying the Lyapunov condition (2.5) with mean $\mu_{i}$ and variance $\sigma_{i}^{2}$. Let $Y_{i}$ be random variables, not necessarily independent such that

$$
E\left[X_{i_{1}} X_{i_{2}} \cdots X_{i_{k}}\right]=E\left[Y_{i_{1}} Y_{i_{2}} \cdots Y_{i_{k}}\right]+O\left(\frac{1}{n}\right)
$$

for $i_{j} \leq f(n)$ for all $1 \leq j \leq k$. Let

$$
s_{n}^{2}=\sum_{i=1}^{f(n)} \sigma_{i}^{2}
$$

Then,

$$
\frac{1}{s_{n}} \sum_{i=1}^{f(n)}\left(Y_{i}-\mu_{i}\right)
$$

converges to the standard normal distribution $N(0,1)$ as $n$ tends to infinity.

Proof. Let $S_{n}=\sum_{j \leq f(n)} X_{j}$ and $T_{n}=\sum_{j \leq f(n)} Y_{j}$. Denote the mean and variance of $S_{n}$ as $c_{n}$ and $s_{n}^{2}$ respectively. As the Lyapunov condition is satisfied for $S_{n}$, by Theorem 2.2, we conclude that as $n$ tends to infinity, $\left(S_{n}-c_{n}\right) / s_{n}$ converges to the standard normal distribution. Since $X_{n}$ 's are bounded, the method of moments applies here and from Theorem 2.1 we have that the $r$-th moment of $\left(S_{n}-c_{n}\right) / s_{n}$ converges to the $r$-th moment of the normal distribution. That is,

$$
m_{r}=\lim _{n \rightarrow \infty} E\left[\left(\frac{S_{n}-c_{n}}{s_{n}}\right)^{r}\right]
$$

for all $r$, where $m_{r}$ denotes the $r$-th moment of the standard normal distribution.

Let $d_{n}$ and $r_{n}^{2}$ denote the mean and variance of $T_{n}$ respectively. By condition (3.6), we have

$$
c_{n}=d_{n}+O(1) \quad \text { and } \quad s_{n}^{2}=r_{n}^{2}+O(1)
$$

as $n$ tends to infinity. Hence, to prove Theorem 3.1, it suffices to show that as $n \rightarrow \infty$

$$
E\left[\left(\frac{S_{n}-c_{n}}{s_{n}}\right)^{r}\right]-E\left[\left(\frac{T_{n}-c_{n}}{s_{n}}\right)^{r}\right] \rightarrow 0
$$

for each $r$. We have

$$
E\left[S_{n}^{r}\right]=\sum_{u=1}^{r} \sum^{\prime} \frac{r !}{r_{1} ! \cdots r_{u} !} \frac{1}{u !} \sum^{\prime \prime} E\left[X_{i_{1}} \cdots X_{i_{u}}\right],
$$

where $\sum^{\prime}$ runs over tuples $\left(r_{1}, \cdots, r_{u}\right)$ satisfying $r_{1}+\cdots+r_{u}=r$ and $\sum^{\prime \prime}$ is over tuples $\left(i_{1}, \cdots, i_{u}\right)$, where $i_{j}$ 's are distinct and not exceeding $f(n)$. 
Similarly, we get

$$
E\left[T_{n}^{r}\right]=\sum_{u=1}^{r} \sum^{\prime} \frac{r !}{r_{1} ! \cdots r_{u} !} \frac{1}{u !} \sum^{\prime \prime} E\left[Y_{i_{1}} \cdots Y_{i_{u}}\right]
$$

where $\sum^{\prime}$ and $\sum^{\prime \prime}$ are as in (3.7). By (3.6), the summands in (3.7) and (3.8) differ by $O(1 / n)$. Hence,

$$
\left|E\left[S_{n}^{r}\right]-E\left[T_{n}^{r}\right]\right| \ll \frac{1}{n}\left(\sum_{j \leq f(n)} 1\right)^{r}=O\left(\frac{f(n)^{r}}{n}\right) .
$$

Now we have

$$
E\left[\left(S_{n}-c_{n}\right)^{r}\right]=\sum_{k=0}^{r}\left(\begin{array}{l}
r \\
k
\end{array}\right) E\left[S_{n}^{k}\right]\left(-c_{n}\right)^{r-k}
$$

Similarly,

$$
E\left[\left(T_{n}-c_{n}\right)^{r}\right]=\sum_{k=0}^{r}\left(\begin{array}{l}
r \\
k
\end{array}\right) E\left[T_{n}^{k}\right]\left(-c_{n}\right)^{r-k}
$$

Comparing these expressions, we get

$$
\left|E\left[\left(S_{n}-c_{n}\right)^{r}\right]-E\left[\left(T_{n}-c_{n}\right)^{r}\right]\right| \ll \sum_{k=0}^{r}\left(\begin{array}{l}
r \\
k
\end{array}\right) \frac{f(n)^{k}}{n} c_{n}^{r-k}=\frac{\left(f(n)+c_{n}\right)^{r}}{n} .
$$

Since $X_{i}$ 's take bounded values, we have $c_{n}=O(f(n))$. Using the condition $\log f(n)=o(\log n)$, we conclude that

$$
\lim _{n \rightarrow \infty} \frac{\left(f(n)+c_{n}\right)^{r}}{n}=0 .
$$

Dividing by $s_{n}^{r}$, we see that $\left(^{*}\right)$ follows.

\section{Proof of Theorem 1.1}

Our method of proof follows Billingsley [Bi69]. For a prime $p$, let

$$
\delta_{p}(m):= \begin{cases}1 & \text { if } p \mid m \\ 0 & \text { otherwise }\end{cases}
$$

Then,

$$
\omega_{y}(m)=\sum_{p \leq y} \delta_{p}(m)
$$

If $p_{1}, p_{2}, \cdots, p_{u}$ are a set of distinct primes $\leq y$, then

$$
P_{n}\left[m \mid \delta_{p_{1}}(m)=\cdots=\delta_{p_{u}}(m)=1\right]=\frac{1}{n}\left[\frac{n}{p_{1} p_{2} \cdots p_{u}}\right],
$$

where $[x]$ denotes the greater integer $\leq x$. This shows that under $P_{n}, \delta_{p_{i}}$ 's behave like independent random variables asymptotically, but are not independent random variables. For a function $f$ of positive integers, define

$$
E_{n}[f]=\frac{1}{n} \sum_{m=1}^{n} f(m) .
$$

For all primes $p$, let $X_{p}$ be independent random variables taking values $\{0,1\}$, satisfying

$$
P\left[X_{p}=1\right]=\frac{1}{p} \quad \text { and } \quad P\left[X_{p}=0\right]=1-\frac{1}{p} .
$$


If $p_{1}, \cdots, p_{u}$ are distinct, then we have

$$
P\left[X_{p_{1}}=\cdots=X_{p_{u}}=1\right]=\frac{1}{p_{1} p_{2} \cdots p_{u}} .
$$

Let $S_{n}=\sum_{p \leq y_{n}} X_{p}$. The mean and variance of $S_{n}$ are given by

$$
c_{n}=\sum_{p \leq y_{n}} \frac{1}{p}=\log \log y_{n}+O(1)
$$

and

$$
s_{n}^{2}=\sum_{p \leq y_{n}} \frac{1}{p}\left(1-\frac{1}{p}\right)=\log \log y_{n}+O(1) .
$$

Since $X_{p}$ 's are independent, we have

$$
E\left[X_{p_{1}} \cdots X_{p_{u}}\right]=\frac{1}{p_{1} \cdots p_{u}} .
$$

Also,

Hence,

$$
E_{n}\left[\delta_{p_{1}} \cdots \delta_{p_{u}}\right]=\frac{1}{n}\left[\frac{n}{p_{1} \cdots p_{u}}\right]
$$

$$
E\left[X_{p_{1}} \cdots X_{p_{u}}\right]-E_{n}\left[\delta_{p_{1}} \cdots \delta_{p_{u}}\right]=\frac{1}{n}\left(\frac{n}{p_{1} \cdots p_{u}}-\left[\frac{n}{p_{1} \cdots p_{u}}\right]\right)=O\left(\frac{1}{n}\right)
$$

for all $p_{i} \leq y_{n}$. The proof now follows from Theorem 3.1.

As an application of this result, we deduce the following corollary.

Corollary 4.1. Suppose $\log \log y_{n}=(\log \log n)(1+o(1))$. With probability 1 , almost all the prime factors of $n$ are less than $y_{n}$. In particular, we can apply this to $y_{n}=n^{1 / \log \log n}$.

\section{Concluding remarks}

The fact that for additive functions $f$ for which an Erdős-Kac type theorem holds, that a localized Erdős-Kac also holds, as in Theorem 1.1, is known and is due to Kubilius, Barban and A.I. Vinogradov (see page 123 of [El79a]). Our contribution here is a new proof of this fact adapting the method of Billingsley [Bi69]. This method is quite versatile and it has wider applications in a very general setting discussed in [MMP]. The relation between distribution of $f_{y_{n}}$ under the measure $P_{n}$ and that of $\sum_{p<y_{n}} X_{p}$, for independent random variables $X_{p}$, taking values $f(p)$ and 0 with probability $1 / p$ and $1-1 / p$ respectively, is captured in the Kubilius Fundamental lemma alluded to above. This lemma states that

$$
P_{n}\left(m: f_{y}(m) \leq z\right)=P\left(\sum_{p \leq y} X_{p} \leq z\right)+O\left(\exp \left(-c \frac{\log n}{\log y}\right)\right)+n^{-\delta} .
$$

From this, one can also derive explicit error terms.

The idea of deriving a central limit theorem in the context of not necessarily independent random variables is also not new. For instance, the notion of $\alpha$-mixing discussed in [Bi79, p. 363] relaxes the notion of independence. A central limit theorem can be derived in such a context. However, our treatment offers an alternate approach to a variation of the central limit theorem suitable for other applications.

Acknowledgement. We thank Siddhi Pathak and the referee for detailed comments on an earlier version of the paper. 


\title{
References
}

[Bi69] P. Billingsley, On the central limit theorem for the prime divisor functions, Amer. Math. Monthly, 76 (1969), 132-139.

[Bi79] P. Billingsley, Probability and measure, Wiley Series in Probability and Mathematical Statistics, John Wiley \& Sons, New York-Chichester-Brisbane, (1979).

[El79a] P.D.T.A. Elliot, Probabilistic number theory. I, Grundlehren der Mathematischen Wissenschaften [Fundamental principles of mathematical science], Vol. 239, Springer-Verlag, New York-Berlin, (1979).

[El79b] P.D.T.A. Elliot, Probabilistic number theory. II, Grundlehren der Mathematischen Wissenschaften [Fundamental principles of mathematical science], Vol. 239, Springer-Verlag, New York-Berlin, (1979).

[EK40] P. Erdős, M. Kac, The Gaussian law of errors in the theory of additive number theoretic functions, Amer. J. Math, 62 (1940), 738-742.

[Hal55] H. Halberstam, On the distribution of additive number theoretic functions, J. London Math. Soc., 30 (1955), 43-53.

[HR1917] G.H. Hardy, S. Ramanujan, The normal number of prime factors of a number n, Quart. J. Pure. Appl. Math., 48 (1917), 323-339.

[MM84a] M.R. Murty, V.K. Murty, Prime divisors of Fourier coefficients of modular forms, Duke Math. J., 51 no. 1, (1984), $57-76$.

[MM84b] M.R. Murty, V.K. Murty, An analogue of the Erdős-Kac theorem for Fourier coefficients of modular forms, Indian J. Pure Appl. Math., 15 no. 10, (1984), 1090-1101.

[MMP] M.R. Murty, V.K. Murty, S. Pujahari An all-purpose Erdős-Kac theorem, to appear.

[Sel53] A. Selberg, Note on a paper by L.G. Sathe, J. Indian Math. Soc., 17 (1953), 83-141.

[Sha56] H.N. Shapiro, Distribution functions of additive arithmetic functions, Proc. Nat. Acad. Sci. U.S.A., 42 (1956), 426430.

[Tu34] P. Turán, On a theorem of Hardy and Ramanujan, J. London Math. Soc, 9 (1934), $274-276$.

\author{
Anup B. Dixit \\ Institute of Mathematical Sciences \\ CIT Campus Taramani \\ Chennai - 600113, INDIA \\ e-mail: anupdixit@imsc.res.in

\section{Ram Murty} \\ Department of Mathematics and Statistics \\ Jeffery Hall, Queen's University, Kingston \\ Canada, ON K7L 3N6 \\ e-mail: murty@mast.queensu.ca
}

